\title{
60 Jahre WHO - von internationaler zu globaler Gesundheit
}

\author{
60 Years of WHO - from International to Global Health
}

\author{
Autor \\ M. Wildner \\ Institut \\ Bayerisches Landesamt für Gesundheit und Lebensmittelsicherheit
}

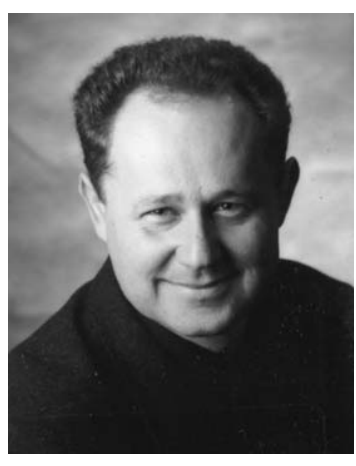

PD Dr. Manfred Wildner
Bibliografie

DOI $10.1055 / \mathrm{s}-2008-1080929$

Gesundheitswesen 2008;

70: 265-266

(c) Georg Thieme Verlag KG

Stuttgart · New York

ISSN 0941-3790

Korrespondenzadresse

\section{PD Dr. M. Wildner}

Bayerisches Landesamt für

Gesundheit und Lebensmittelsicherheit

Veterinärstr. 2

85762 Oberschleissheim

manfred.wildner@lgl.bayern.de
Der Weltgesundheitstag am 7. April eines jeden Jahres markiert den Gründungstag der Weltgesundheitsorganisation (WHO) - dieses Jahr den „60. Geburtstag“. Am 7. April 1948 wurde die Gründung der WHO als eigenständige internationale Gesundheitsorganisation wirksam, die bereits von der Konferenz der Vereinten Nationen (UN) in San Francisco im Jahr 1945 einstimmig beschlossen worden war. Grund zum Feiern? Allemal - die WHO hat aus diesem Anlass auch eine ansehnliche Chronologie Ihrer Erfolge im Internet verfügbar gemacht hat („Full chronology of public health milestones“, [1]).

Beispiele dieser Meilensteine? Traditionell stehen die Infektionskrankheiten im Fokus: Die Betreuung und Weiterentwicklung der Internationalen Gesundheitsvorschriften, Forschungs- und Trainingsprogramme zu Tropenkrankheiten, die Tuberkulose-Impfung mit BCG und das erweiterte Impfprogramm, die kostenfreie Übernahme der Rechte für die Polio-Vakzine von Dr. Albert Sabin und ihr weltweiter Einsatz (1974), die erfolgreiche Bekämpfung der Frambösie, die weltweite Ausrottung der Pocken (1958-1979), die Programme mit dem Ziel einer weltweiten Eradikation der Poliomyelitis (1988), der Lepra (1991), der Dragunculiasis (1995), der Masern (2001), Malaria-, HIV/AIDS, Tuberkulose-Bekämpfungsprogramme, die Kontrolle der SARS-Epidemie 2003 u.a.m..

Weniger spektakulär, doch von gleichrangiger Bedeutung waren und sind die Aktionen bezogen auf die nicht-übertragbaren Krankheiten: Die Betreuung und Weiterentwicklung der Internationalen Klassifikation der Krankheiten und Todesursachen (ICD) und anderer Klassifikationen wie der ICF als wichtige Steuerungsinstrumente, der erste Bericht zu Diabetes mellitus (1965), die Gründung des Internationalen Krebsforschungsinstituts in Lyon (1965), die Betonung der primären Gesundheitsversorgung mit Integration der Rehabilitation (Beschluss der World Health
Assembly 1976, „Gesundheit für alle“ und Alma Ata 1978), die Betonung von Prävention und Gesundheitsförderung (Ottawa 1986 und ihre Folgekonferenzen), die Entschlüsse zur Nicht-Diskriminierung von AIDS-Kranken (1988), die Bildung der Kommissionen zu makroökonomischen und sozialen Determinanten der Gesundheit (2000 und 2005), der erste Weltgesundheitsbericht zu Gewalt (2002), das Anti-Tabak-Rahmenübereinkommen (2003) und der WHO-Bericht zur Globalen Tabakepidemie (Report on the Global Tobacco Epidemic, 2008), Klima und Gesundheit - die Liste ist wiederum nicht abschließend. Eine Liste „technischer“ Erfolge, erreicht durch die Arbeit einer internationalen Vermittlungsstelle? Hier kann nur ein „Ja mit Einschränkungen“ gesagt werden. Eine Stärke der Arbeitsweise der WHO ist gerade ihre indirekte Einflussnahme durch weitgehend unverbindliche Fachempfehlungen - auf den ersten Blick ein Paradox. Auf den zweiten Blick ein gutes Beispiel für demokratische Einflussnahme in einer pluralen Welt, die auch Mut verlangt. Diesen Mut zeigt auch das Mitgliedsland Deutschland als wichtiger Beitragszahler und Unterstützer spezifischer Initiativen für die WHO als ganzes und im regionalen europäischen Kontext.

Eine zweite wichtige Funktion der WHO ist ihre „Leadership“-Rolle: schon in ihrer Verfassung darauf hinzuweisen und trotz Widerständen daran festzuhalten, dass Gesundheit eben mehr ist als die „Abwesenheit von Krankheit“. Dass das Recht auf Leben und Gesundheit ein fundamentales Menschenrecht ist, das es grenzüberschreitend zu bewahren und oftmals zu erkämpfen gilt - für alle Menschen und bisweilen gegen andersartige Interessen [2,3]. Welcher Natur diese andersartigen Interessen sind? Machterhalt z. B. in Diktaturen und sozialen (Kasten-)Strukturen, Ignoranz, Hass und Diskriminierung, fehlgeleitete wirtschaftliche Interessen - auch Angst: Vor dem eigenen Menschsein und dem der anderen in 
einem „globalen Dorf“. Auf den allmählichen Übergang von einem „internationalen“, also zwischenstaatlichen Verständnis zu einem „globalen“, also staatenübergreifenden Verständnis von Gesundheit in der Arbeit der WHO sei hingewiesen [4]. Gesundheit ist spätestens im 21. Jahrhundert geprägt von zunehmenden wirtschaftlichen, sozialen, politischen und kulturellen Verflechtungen. Und damit von gemeinsamen Gefährdungen, Herausforderungen und Chancen hinsichtlich der Gesundheit in der von Menschen gemachten und in der natürlichen Umwelt. Das Heil von einer Institution zu erwarten, die sicher auch ihre Problematiken in sich trägt, wäre naiv. Vielleicht sollte daher der Geburtstagswunsch an uns alle gerichtet werden: Weiterzuarbeiten an einer „Gesundheit für alle“, frei von Gier, Hass und Angst.

\section{Literatur}

1 http://www.who.int/features/history/WHO_60th_anniversary_chro nology.pdf, Zugriff am 15.05.2008

2 http://www.who.int/governance/eb/who_constitution_en.pdf, Zugriff am 15.05.2008

3 http://www.who.int/tobacco/mpower/en/index.html, Zugriff am 15.05.2008

4 Fee E, Cueto M, Brown TM. WHO at 60: snapshots from its first six decades. Am J Public Health 2008; 98: 630-633 